\title{
A Deceptive Color-Doppler Image After Endovascular Aorta Repairing
}

Enrico Giustiniano* and Nadia Ruggieri

Humanitas Research Hospital, Department of Anesthesia and Intensive Care, Italy

Received: November 13, 2014; Accepted: March 17, 2015, Published: March 27, 2015

*Corresponding author: Enrico Giustiniano, Humanitas Research Hospital, Department of Anesthesia and Intensive Care, Italy, Pin: 20089; E-mail: enrico.giustiniano@gmail.com

\section{Backgrounds}

Trans-Esophageal Echocardiography (TEE) is a tool that is increasingly employed in the operative theater, and also used during cardiac surgery operations. TEE has an important role during Thoracic Endovascular Aortic Repair (TEVAR) too, as it provides immediate detection of endo-leak and its management. $[1,2]$.

We report a case of a misleading image of a false type1-endoleak by TEE at the end of a thoracic endoprosthesis deployment which was not resolved by endoaortic balloon inflation.

\section{Case Report}

We report a case of TEVAR in a male patient aged 69 years, ASA 3, performed in general anesthesia induced with Fentanyl $1 \mathrm{mcg} / \mathrm{kg}$ i.v + Propofol $2 \mathrm{mg} / \mathrm{kg}$ i.v. An oral tracheal tube was inserted after myorelaxation with cis-Atracurium $10 \mathrm{mg}$ i.v bolus and further administration of $0.15 \mathrm{mg} / \mathrm{kg}$ every 30 minutes. Anesthesia continued with administration of a gas mixture of air and oxygen $\left(\mathrm{FiO}_{2}\right.$ 0.5-0.6, according Blood Gas-Analysis (BGA) results) and Sevoflurane 1-2\%. Mechanical ventilation setting: Tidal Volume $7 \mathrm{ml} / \mathrm{kg}$; respiratory rate 12 apm and Positive EndExpiratory Pressure (PEEP) 5-7 $\mathrm{cm} \mathrm{H}_{2} \mathrm{O}$.

Intraoperative monitoring included electrocardiogram (D2 and V5) and ST line analysis, Heart Rate (HR), Non-Invasive Blood Pressure (NIBP) and Invasive Blood Pressure (IBP) after right radial artery line insertion connected with FloTrac/ Vigileo $^{\mathrm{TM}}$ sensor (Edwards Lifesciences, Irvine, USA) for Cardiac Output (CO; Cardiac Index, CI) monitoring, peripheral oxygen saturation $\left(\mathrm{SpO}_{2}\right)$, Central Venous Pressure (CVP), End-Tidal Carbon Dioxide $\left(\mathrm{EtCO}_{2}\right)$ and diuresis. A subarachnoid catheter was inserted (Lumbar Drainage Catheter $46 \mathrm{~cm}$ - Codman and Shurtleff, Inc., Reynham, MA, USA) at lumbar level L3-L4 and connected to standard multichannel patient monitoring system to measure intrathecal pressure $\left(\mathrm{P}_{\mathrm{Lq}}\right)$ and withdraw CerebroSpinal Fluid (CSF) to lower it when $\mathrm{P}_{\mathrm{Lq}}>15 \mathrm{mmHg}$. Transesophageal echocardiogram (Squarq Ultrasound System; Probe X7-2t Transesophageal Adult Echo; Philips Healthcare, Bothell,
WA, USA) was used to value cardiac performance, but particularly to monitor the endoprosthesis release and its "landing" on aortic wall. BGA was tested when considered appropriate.

Postoperative analgesia consisted of Paracetamol $1 \mathrm{~g}$ i.v bolus every 6 hours and wound infiltration with L-Bupivacaine $3.75 \mathrm{mg} / \mathrm{ml}$ (20 $\mathrm{ml})$ before cutaneous suturing.

Figure 1 shows descending thoracic aorta before and after endoprosthesis deployment. The image after aorta repair does not show any endoleakage. In figure 2 regarding the proximal end of the endoprosthesis, we observed a turbulent blood flow that did look like endoleak.

Although the greater sensitivity of TEE vs Angiography about small leakage detection, further angiograms were acquired before patient's awakening and they always resulted negative. Given the persistence of the endoleak-like ultrasound imaging, surgeon performed endo-aortic balloon inflation over the proximal end of the prosthesis, but it was unsuccessful.

Then we decided to monitor the patient with further CT-scan postoperatively. The follow-up was uneventful and the patient was discharged from hospital after 3 days. Patient underwent CTscan exam after three and five months after the operation and it was always negative for endoleak or other complications [Figure 3].

The exam was performed after three months from the operation (A-B) and two months later: it never showed any endoleak

\section{Discussion}

Thoracic endovascular aortic repair is currently the firstchoice procedure for thoracic aorta surgery, due to its lower invasiveness compared with traditional operation that makes such surgery the most suitable for high-risk profile patients. Given such advantage, some drawback may occur and complicate the outcome of the procedure, particularly when it cannot be disclosed precociously.

The most frequent adverse event is endoleakage's appearence that can manifest in different types [3]. 


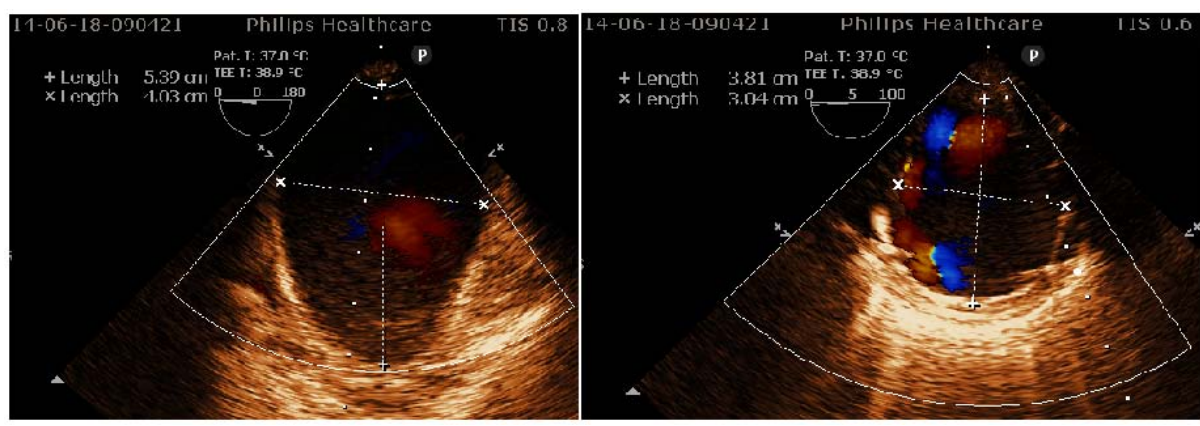

Figure 1: Before and after endoprosthesis deployment.

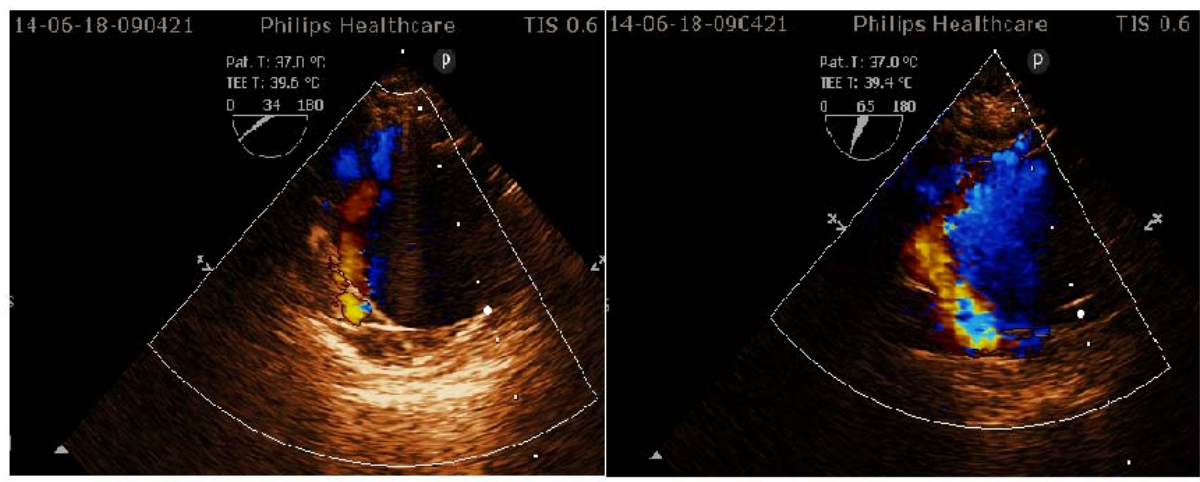

Figure 2: Echo-color-Doppler images.

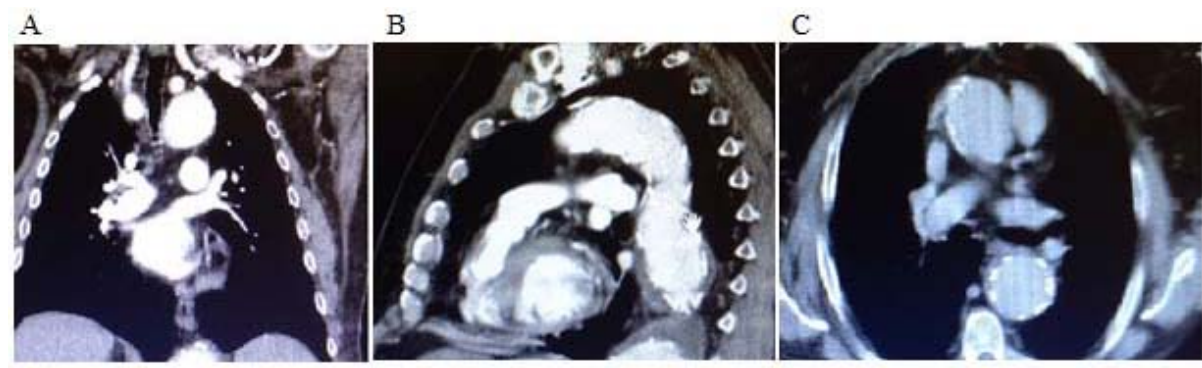

Figure 3: Echo-color-Doppler images.

When endoleak occurs, its correction is generally attempted by endo-aortic balloon inflation and when it does not result decisive further endoprosthesis deployments is the way, or open surgery correction when appropriate [4-5].

In our case, the color-Doppler of TEE showed some blood flow between the aortic wall and the proximal end of the endoprosthesis. The image was persistent also after endoaortic balloon inflation. A second prosthesis would had been contraindicated because the proximal end of the inserted endoprosthesis was close to the emergence of the left common carotid artery. Then, as the angiography did not show any abnormality of the contrast medium flow at the same point where TEE showed the suspected image, we decided to end the procedure considering the color-Doppler picture could be due to the turbulent blood flow through the mesh of the uncovered part of the prosthesis, which was not adjusted with the curvature of the aortic arch.

In any case, given the small dimension of the color-Doppler image of suspected blood-flow, it is known that a little endoleak can close off spontaneously. The follow-up of the patient was uneventful and angio-CT scan control resulted negative for endoleak.

In our opinion, the deceptive image of TEE occurred because of the misalignment between the uncovered proximal end of the endoprosthesis and the internal curve of the aortic arch where the prosthesis did not "land" completely.

We considered that such a drawback may be worthy of notice, as it may often occur, given the tortuousness of the aorta not only at the portion of the arch. It could be misinterpreted, making 
possible un-necessary further insertion of endoprosthesis or open surgery. It may be worthy of attention and stimulate a technological upgrading by industries to create new devices that can solve the issue.

\section{References}

1. Evangelista A, Flachskampf FA, Erbel R, Antonini-Canterin F, Vlachopolos C, Rocchi G, et al. Echocardiography in aortic disease: EAE recommendation for clinical practice. Eur J Echocardiogr. 2010; 11(8): 645-58. doi: 10.1093/ejechocard/jeq056.

2. Fattori R, Caldarera I, Rapezzi C, Rocchi G, Napoli G, Parlapiano M, et al Primary endoleakage in endovascular treatment of the thoracic aorta: importance of intraoperative transesophageal echocardiography. J Thrac Cardiovasc Surg. 2000; 120(3): 490-5.
3. Veith FJ, Baum RA, Ohki T, Amor M, Adiseshiah M, Blankensteijn JD, et al. Nature and significance of endoleaks and endotension: summary of opinions expressed at an international conference. J Vasc Surg. 2002; 35(5): 1029-35.

4. Swaminathan M, Meckensen GB, Podgoreanu MV, McCann RL, Mathew JP, Hughes GC. Spontaneous echocardiographic contrast indicating successful endoleak management. Anesth Analg. 2007; 104(5): 103739.

5. Trimarchi S, Segreti S, Grassi V, Lomazzi C, De Vincenti C, Rampoldi V. Emergent treatment of aortic rupture in acute type B dissection. Ann Cardiothorac Surg. 2014; 3(3): 319-24. doi: 10.3978/j.issn.2225319X.2014.05.05. 